\title{
La gestión directiva o el liderazgo educativo en tiempos de pandemia
}

\author{
Directive management and/or educational leadership in pandemic \\ time.
}

Gestão e/ou liderança educacional em tempos de pandemia.

\author{
Gladys Kochen \\ Investigadora independiente \\ Buenos Aires, Argentina \\ glakochen@gmail.com \\ https://orcid.org/0000-0003-0403-0170
}

Recibido - Received - Recebido: 30/11/2020 Aceptado - Accepted - Aprovado: 03/12/2020

DOI: https://doi.org/10.22458/ie.v22i33.3349

URL: https://revistas.uned.ac.cr/index.php/innovaciones/article/view/3349

\begin{abstract}
Resumen: La situación de emergencia en el mundo vino a interpelar las políticas públicas ¿cómo volver a la escuela?, ¿cómo será volver a una nueva "normalidad"? Los directores tuvieron que comenzar a trabajar con los docentes y alumnos que estaban en sus casas y desde sus casas, haciendo un seguimiento de sus planes, definiendo los problemas para buscar soluciones $y$, sobre todo, redefiniendo la construcción de políticas intersectoriales. Experiencia que evidentemente no debemos perder. Se debe planificar evaluando el sostener y mantener la cercanía durante y después de la pandemia, planificando la vuelta de quienes eventualmente han quedado fuera del sistema, garantizando la incorporación de tecnologías a la comunidad educativa, con participación de docentes y familia. Planificar es necesario, pero también lo es el considerar si lo hacemos teniendo en cuenta la experiencia o simplemente volveremos a planificar para una supuesta "normalidad".
\end{abstract}

Palabras clave: Planificación educativa; emergencia sanitaria; ODS 4; nueva normalidad; pospandemia; liderazgo, COVID-19.

\begin{abstract}
The world's emergency situation came to question public policies: How are we going back to school? What is the new normalcy? Therefore, headteachers had to work along with teachers and students who were home and from home. The work implied a follow-up plan, tracking down problems, finding solutions, and above all, redefining the construction of intersectoral policies. This is, evidently, an experience that we cannot afford to forget. Educational planning should be carried out while evaluating, sustaining, and maintaining proximity during and after the pandemic. The return of those who have eventually been left behind shall be considered as well, in order to guarantee the incorporation of technologies into the educational community, with the participation of teachers and
\end{abstract}


families. Planning is necessary. However, it is also necessary to consider whether we do it considering our previous experiences, or we will re-plan for a supposed "normalcy."

Keywords: educational planning, public health emergency, new normal, post-pandemic, leadership

Resumo: A situação de emergência no mundo, veio para questionar as políticas públicas Como voltar à escola? Como será o retorno a uma nova "normalidade"? Os diretores tiveram que começar a trabalhar com professores e alunos que estavam em casa e de suas casas, acompanhando seus planos, definindo problemasa fim de buscar soluções e, sobretudo, redefinindo a construção de políticas intersetoriais. Esta é uma experiência que obviamente não deve ser perdida. Devemos planejar avaliando como sustentar e manter a proximidade durante e após a pandemia. Planejamento para o retorno daqueles que eventualmente foram deixados fora do sistema. Garantir a incorporação de tecnologias na comunidade educacional, com a participação de professores e famílias. O planejamento é necessário. Mas também é necessário considerar se o fazemos à luz da experiência ou simplesmente replanejamos para uma suposta "nomalidade".

Palavras-chave: Planejamento educacional, emergência sanitária, nova normalidade, pós-pandemia, liderança. 


\section{INTRODUCCIÓN}

La historia reciente y vivida en el año 2020 nos muestra que el tiempo y el contexto van marcando la letra y el camino. Si este artículo me lo hubieran solicitado solamente un año atrás, me hubiera enfocado en explicitar aspectos relativos a saberes previos, el rol del director en las instituciones educativas o los diferentes modelos de liderazgo y gestión que se observan en las mismas. Es probable que me hubiera detenido a precisar el por qué es necesario fortalecer el rol del director y su lugar clave en la organización, la administración, la dimensión pedagógico-didáctica, la relación con la comunidad de la escuela, y su responsabilidad en el logro de un clima educativo inclusivo que cuide las trayectorias educativas de las personas estudiantes y garantice una educación de calidad.

Sin embargo, hoy, si bien podría seguir retomando varios de los temas citados, es necesario mirar, profundizar y revisar los argumentos con una lupa diferente. Una lupa que seguramente aumentará varios de los problemas preexistentes, pero que a la luz del 2020 han quedado más visibles. En ese sentido, ¿podremos hacernos cargo de que el año 2020 no existió? ¿Podremos insistir en repetir prácticas anteriores sin aprovechar el momento de crisis generado para revisar y aprender de las lecciones aprendidas en estos tiempos?

Por estos días, el Papa Francisco publicó una nueva encíclica donde plantea lo siguiente: "Si alguien cree que solo se trataba de hacer funcionar mejor lo que ya hacíamos, o que el único mensaje es que debemos mejorar los sistemas y las reglas ya existentes, está negando la realidad". Sencillo de entender, ¿verdad? No se puede hacer funcionar mejor lo que había, ni pretender mejorar el sistema económico, político y educativo existente que en el marco de la pandemia dejó expuestas sus falencias. La situación de emergencia en el mundo vino a interpelar las políticas públicas, en particular las educativas, nos encontramos frente a graves incertidumbres respecto a sus consecuencias: ¿cómo volver a la escuela?, ¿cómo será volver a una supuesta "normalidad"?, ¿quiénes quizá ya no volverán?, entre otras. La preocupación que circula es cómo volver a la escuela, cómo armar protocolos seguros para el regreso y la revinculación de las personas estudiantes.

\section{DESARROLLO}

Lo primero que debemos preguntarnos es ¿a cuál normalidad queremos volver? O formalmente, ¿cuáles son nuestros objetivos prioritarios en términos de política educativa?, ¿cómo se articulan en el espacio de la micropolítica territorial y el aula? Es necesario que cada uno, y también en conjunto, podamos hacer un ejercicio introspectivo para analizar cuál era el foco de nuestras prácticas educativas en los últimos tiempos y cuáles deberían haber sido para que nuestras respuestas fueran adecuadas en la situación de emergencia; es decir, repensar lo que hacíamos para ver qué debemos hacer.

Es necesario que hoy establezcamos más preguntas que afirmaciones, porque si desnaturalizamos lo naturalizado, si deconstruimos los absolutos, podremos aprovechar la experiencia para darle un giro a nuestro trabajo y efectivamente lograr cumplir los derechos a la educación igualitaria. Nos encontramos atravesando una crisis casi sin antecedentes; sin embargo, la humanidad vive en una crisis permanente, en especial América Latina que es el continente con mayor desigualdad en el mundo. En una nota periodística reciente (ver nota 1), leí que las 2100 personas más ricas del planeta poseen más que $60 \%$ de la población mundial (4600 millones de personas). Por tanto, ¿cuáles consecuencias implica la concentración de riqueza en los sistemas educativos?, ¿puede el sistema educativo o el director de una escuela, quien representa uno de los espacios privilegiados del territorio social, cumplir con el objetivo de ofrecer a los sujetos igualdad de oportunidades y movilidad social en ese marco? La respuesta la dejo abierta. 
Reflexionando sobre la cita del Papa de nuestra introducción, ¿será esto normal para la humanidad? El tema y el problema exceden al campo educativo. Es indispensable pensar la educación en el marco de un proyecto político-económico en el que, por un lado, se ponga el foco en la redistribución de la riqueza financiera que, de acuerdo con los datos expuestos, no para de concentrarse, pero que junto con ello se trabaje fuertemente en la redistribución del conocimiento y la tecnología, que hoy más que nunca, se han erigido como una verdadera e importante riqueza para la humanidad.

En el año 2015, 193 países miembros de las Naciones Unidas reunidos en Chile firmaron un acuerdo de compromiso consensuado por todos para el cumplimiento de la Agenda 2030 que incluía 17 objetivos de desarrollo sostenible (ODS). En Educación, el ODS 4 específicamente planteó "garantizar una educación inclusiva, equitativa y de calidad y promover oportunidades de aprendizaje durante toda la vida para todas las personas". Si bien, aún nos encontramos lejos de su cumplimiento, podríamos decir que este objetivo ha servido como una guía para definir el camino de casi todos los países de la región. La pandemia ha modificado prioridades, pero en cierto modo ha reafirmado la necesidad de su cumplimiento. El objetivo considera la educación como un bien público y plantea centralmente la necesidad de hacer cumplir el derecho a la educación redistribuyendo el conocimiento de manera equitativa y asegurando su calidad.

La emergencia sanitaria ha significado, para los ministerios de educación de la región, un desafío sin precedentes y con el propósito de prevenir la propagación del coronavirus y mitigar su impacto se tomó, casi en todos los países, la decisión de cerrar masivamente las instituciones educativas. A raíz de lo anterior, millones de estudiantes vieron interrumpidos o afectados sus procesos de aprendizaje (ver nota 2). Al intercambiar experiencias con colegas de la región, lo primero que surge es que la pandemia interrumpió las planificaciones para el cumplimiento del ODS 4, lo cual podría ser entendible, ya que la emergencia sanitaria se impuso como un enemigo invisible que amenaza la vida, que definió nuevas prioridades, y que frenó todo lo que se venía desarrollando.

En el contexto de la pandemia, cada país intentó construir diferentes estrategias como respuesta frente a la crisis, con el fin de cumplir con la continuidad educativa. Entregas de computadoras para los sectores más vulnerables, programas de educación a distancia diferenciadas por niveles, entregas de libros de textos, planes de lecturas para las familias, programas televisivos educativos, materiales específicos para apoyar la crianza en las casas, juegotecas familiares para los más pequeños, encuentros virtuales con las familias, clases por zoom, entre otras.

Han sido los directores de las instituciones educativas junto a los docentes los encargados de hacer llegar estas políticas a la comunidad; por tanto, ¿volver a la supuesta "normalidad" implicará perder estas estrategias?, ¿no son algunas ellas muy eficaces para contribuir al cumplimiento del ODS 4?; es decir una política educativa inclusiva, equitativa y de calidad. Muchos directores tuvieron que comenzar a monitorear el trabajo de los docentes que estaban en sus casas y, también desde sus casas, seguir sus planes, dialogar con todos, definir los problemas para buscar soluciones y, sobre todo, debieron aprender a construir políticas intersectoriales. Otros que ya lo venían haciendo, profundizaron sus esfuerzos. Es una experiencia que evidentemente no debemos perder.

En abril del 2020, la reconocida pedagoga Inés Dussel (2020) realizó un conversatorio organizado por el ISEP, Ilamado "la clase en pantuflas" y en su discurso se cuestionaba algunas preguntas que me parecieron interesantes: ¿qué cambia en la clase cuando estamos en casa y los chicos en la suya?, ¿cuál tipo de clase es esa?, ¿qué pasa con nuestras pedagogías, con nuestro trabajo docente? En general, se analiza la importancia de mantener la escuela o la universidad "a distancia", ya sea a través de plataformas digitales, programas televisivos o por medios materiales impresos. Para algunos, este escenario anticipa lo que será la educación del futuro, de aprendizajes ubicuos, sin espacios escolares específicos y sin tiempos programados ("justo-a-medida" del usuario). 
Se plantea que el futuro es hoy, que hoy comienza el nuevo paradigma que demostrará que la escuela es una institución obsoleta y los maestros molestan en el acceso directo de niños y jóvenes al conocimiento. No obstante, también se propone una visión opuesta, la del escenario distópico, parecido al "sálvese quien pueda", que llevará a la pérdida de la dimensión pública y común de la escolarización y tendrá graves consecuencias en sociedades profundamente desiguales, porque unos acceden a plataformas y acompañamiento de primer nivel y otros tienen que arreglárselas con pocos impresos o charlas por WhatsApp.

A partir de las palabras de Dussel (2020), apreciamos que, en la primera postura, resulta preocupante ver que la figura del docente tiende a desaparecer $y$, como bien señala la pedagoga, parece ser alguien que estorba en el proceso del conocimiento. En la segunda postura, la preocupación está centrada en la desigualdad que puede generar la pérdida de la presencialidad escolar en los alumnos por problemas en el acceso a internet, se deja implícita la importancia del docente como garantía del derecho igualitario a la educación. Creo que este es un punto importante por destacar y en el contexto de la pandemia se ha hecho más evidente. La pregunta que aparece es justamente si el rol del director deberá cambiar a partir de este año o cuáles son sus nuevos desafíos en este contexto.

Recientemente, UNICEF (2020) Argentina ha propuesto un documento para pensar en los nuevos desafíos para el equipo de conducción en el marco de la pandemia (ver nota 3). Creo que es un documento importante de tener en cuenta pues responde a una serie de preguntas formuladas en términos de desafíos y ofrece algunas propuestas que pueden servir como apoyo. Básicamente, el documento reflexiona acerca de la necesidad de que el director planifique con sus equipos docentes de acuerdo con la realidad de su comunidad educativa, y con la sensibilidad necesaria para encontrar las estrategias que puedan mitigar los daños ocasionados por la pandemia, así como preparar y prevenir para lo que pueda ocurrir en un futuro.

Sin lugar a duda, las experiencias vividas en este difícil tiempo nos han enseñado mucho, pero es necesario capitalizar las lecciones aprendidas, convirtiéndolas en oportunidades que no debemos desaprovechar.

\section{ALGUNAS CONCLUSIONES}

Como se señaló, las crisis generadas por emergencias o desastres suelen impactar en los sistemas educativos de numerosas y variadas maneras. En todos los casos, siempre reducen la capacidad de los sistemas educativos para brindar una educación continua, inclusiva, equitativa y de calidad para todas las personas, ya que las consecuencias tienden a afectar con mayor intensidad a las poblaciones vulnerables y las desigualdades en la educación pueden profundizarse. Por tanto, se requiere una reacción planificada del sistema educativo.

Planificar es un acto técnico-político que implica contar con un conjunto de herramientas y una intencionalidad política clara para el logro de las transformaciones sociales deseables para la sociedad, no es un simple acto burocrático. Tonucci propone que en lugar de pensar todo lo que perdieron las escuelas, los docentes y especialmente los alumnos y alumnas en sus aprendizajes a causa de la no presencialidad, se debería evaluar lo que la experiencia justamente hizo aprender. Hay que animarse y dar vuelta la moneda (Martin, 2020).

Muchos directores han acompañado esta situación y han llevado a cabo acciones novedosas: estar cerca de la comunidad educativa, cerca de las familias, consolar a niños y niñas que han perdido familiares, revisar con los docentes nuevas estrategias. Otros, por el contrario, las han continuado haciendo. Lo cierto es que en la "nueva normalidad" no debemos perder lo ganado porque entonces habremos perdido una oportunidad de transformación social y educativa. Sin lugar a duda, el rol del director es complejo por la diversidad de funciones que abarca, hace malabares entre las normas y la realidad, como muchas veces señala la educadora Sandra Nicastro. 
Me gustaría finalizar compartiendo una reflexión de Carlos Mattus (1987): "Si planificar es sinónimo de conducir conscientemente, o planificamos o somos esclavos de la circunstancia, lo cual puede llevarnos a perder la posibilidad de elegir un futuro más justo". Planificar es necesario, pero o planificamos considerando la experiencia y las lecciones aprendidas o perdemos la oportunidad y solo volvemos a planificar para el retorno a la "normalidad".

\section{Notas:}

1. Seibt, S. (2020, 8 de octubre). ¿Cómo se enriquecieron los millonarios del mundo durante la pandemia? France 24. Recuperado de: https://www.france24.com/es/20201008-enriquecimiento-millonarios-pandemia.

2. Más información en: https://es.unesco.org/covid19

3. Más información en: https://www.unicef.org/argentina/media/8381/file/Serie-Conduccion-Covid-1.pdf

\section{REFERENCIAS}

Dussel, I. (2020). La clase en pantuflas. En Dussel, I., Ferrante, P. y Pulfer, D. (comp.) (2020). Pensar la educación en tiempos de pandemia: entre la emergencia, el compromiso y la espera. Ciudad Autónoma de Buenos Aires: UNIPE: Editorial Universitaria, 2020. Recuperado de: https://editorial.unipe.edu. ar/colecciones/politicas-educativas/pensar-la-educaci\%C3\%B3n-en-tiempos-de-pandemia-entrela-emergencia,-el-compromiso-y-la-espera-detail

Martin, J. (2020). Coronavirus. Franceso Tonucci: Hablamos mucho de los niños, pero poco con los niños. RTVE. Recuperado de: https://www.rtve.es/noticias/20200829/entrevista-pedagogo-investigadoritaliano-francesco-tonucci/2041015.shtml

Mattus, C. (1987). Política, Planificación y Gobierno. Buenos Aires: Organización Panamericana de la Salud. Recuperado de: http://www.bnm.me.gov.ar/giga1/documentos/EL001185.pdf

Seibt, S. (2020). ¿Cómo se enriquecieron los millonarios del mundo durante la pandemia? France 24. Recuperado de: https://www.france24.com/es/20201008-enriquecimiento-millonarios-pandemia

UNESCO (2020). La educación en América Latina y el Caribe ante la COVID-19. Recuperado de: https:// es.unesco.org/fieldoffice/santiago/covid-19-education-alc

UNICEF (2020). Serie: "Los equipos de conducción frente al Covid-19: claves para acompañar y orientar a los docentes, las familias y los estudiantes en contextos de emergencia". Recuperado de: https://www. unicef.org/argentina/publicaciones-y-datos/serie-conduccion-covid

\section{(c) $(1) \Theta$}

